\title{
Ontologies, Mental Disorders and Prototypes
}

\author{
M. Cristina Amoretti ${ }^{1}$, Marcello Frixione ${ }^{1}$, Antonio Lieto ${ }^{2}$, and Greta Adamo ${ }^{3}$ \\ ${ }^{1}$ DAFIST, Philosophy Section, University of Genoa, Italy \\ ${ }^{2}$ Department of Computer Science, University of Turin, Italy and ICAR-CNR, Italy \\ ${ }^{3}$ Bruno Kessler Foundation, Trento, Italy
}

\begin{abstract}
As it emerged from philosophical analyses and cognitive research, most concepts exhibit typicality effects, and resist to the efforts of defining them in terms of necessary and sufficient conditions. This holds also in the case of many medical concepts. This is a problem for the design of computer science ontologies, since knowledge representation formalisms commonly adopted in this field (such as, in the first place, the Web Ontology Language - OWL) do not allow for the representation of concepts in terms of typical traits. However, the need of representing concepts in terms of typical traits concerns almost every domain of real world knowledge, including medical domains. In particular, in this article we take into account the domain of mental disorders, starting from the DSM- 5 descriptions of some specific mental disorders. On this respect, we favor a hybrid approach to the representation of psychiatric concepts, in which ontology oriented formalisms are combined to a geometric representation of knowledge based on conceptual spaces.
\end{abstract}

Keywords: Representation of concepts, Formal ontologies, Conceptual spaces, Medical ontologies, Mental Disorders, DSM-5

\section{Introduction}

As it emerged from philosophical analyses and cognitive research, most concepts exhibit typicality effects, and resist to the efforts of defining them in terms of necessary and sufficient conditions. This holds also in the case of many medical concepts. This is a problem for the design of computer science ontologies, since knowledge representation formalisms commonly adopted in this field (such as, in the first place, the Web Ontology Language - OWL) do not allow 
for the representation of concepts in terms of typical traits. However, the need of representing concepts in terms of typical traits concerns almost every domain of real world knowledge, including medical domains. In this article we take into account the domain of mental disorders, starting from the DSM-5 descriptions of some specific mental disorders. On this respect, we favor a hybrid approach to the representation of psychiatric concepts, in which ontology oriented formalisms are combined to a geometric representation of knowledge based on conceptual spaces.

In Section 1. we shall expose some problems faced by the classical theory of concepts, according to which concepts can be defined through necessary and sufficient conditions. In particular, we shall examine the important issues raised by conceptual "typicality", which concerns both common-sense and medical concepts, focusing on the general concept of MENTAL DISORDER and the various concepts of individual mental disorders as described by DSM-5. In Section 2 . we shall briefly summarize the most common way to deal with the problem of concept representation, which received a great deal of attention within the field of artificial intelligence (AI), due to its relevance for semantic technologies and for the development of formal ontologies. We shall maintain that the most representative formalisms currently adopted for the development of formal ontologies, known as description logics (DLs), are unfortunately unable to represent concepts in prototypical terms. In Section 3. we shall describe an ontology we specifically build to represent the general concept of MENTAL DISORDER and (most of) the various concepts of individual mental disorders. Despite the fact that there already are formal ontologies dealing with mental disorders, we decided to develop a new one trying to overcome some of their potential limitations. As our formal ontology, despite being more DSM-5 compliant than others, is still unable to handle typicality effects, in Section 4. we shall propose a hybrid approach combining a "classical" component (in which concepts are represented in terms of necessary and/or sufficient conditions) with a "typicality-oriented" component, allowing both prototype and exemplar-based representations. 


\section{Representing Concepts: Some Problems Raised by Medicine}

In philosophy and cognitive sciences, different theories about the nature of concepts have been proposed. According to the traditional view, known as "classical", concepts can be simply defined in terms of sets of necessary and sufficient conditions. This theory was dominant since the times of Aristotle until the mid'70s of the last century, when the philosophical analyses by Ludwig Wittgenstein (Wittgenstein 1953) and the experimental results obtained by Eleaonor Rosch (Rosch 1975; Rosch and Mervis 1975) showed that, for most of the common-sense concepts, this position does not hold since conceptual structures are mainly characterized by "typical" category membership cues and thus suggested that are organized in human mind in terms of prototypes. Since then, different positions and theories on the nature of concepts have been proposed in order to explain the aspects concerning conceptual "typicality". Usually, they are grouped in three main classes, namely: prototype views, exemplar views and theory-theories (see e.g. Murphy 2002; Machery 2009). All of them are assumed to account for (some aspects of) prototypical effects in conceptualization.

According to the prototype view (introduced by Rosch), knowledge about categories is stored in terms of prototypes, i.e. in terms of some representation of the "best" instances of the category. For example, the concept CAT should coincide with a representation of a prototypical cat. In the simpler versions of this approach, prototypes are represented as (possibly weighted) lists of features.

According to the exemplar view, a given category is mentally represented as a set of specific exemplars explicitly stored within memory: the mental representation of the concept CAT is the set of the representations of (some of) the cats we encountered during our lifetime.

Theory-theory approaches adopt some form of holistic point of view about concepts. According to some versions of the theory-theories, concepts are analogous to theoretical terms in a scientific theory. For example, the concept CAT is individuated by the role it plays in our mental theory of zoology. In other version of the approach, concepts themselves are identified with micro-theories of some sort. For ex- 
ample, the concept CAT should be identified with a mentally represented micro-theory about cats.

These approaches turned out to be not mutually exclusive. Rather, they seem to succeed in explaining different classes of cognitive phenomena, and many researchers hold that all of them are needed to explain psychological data (see again Murphy 2002; Machery 2009).

The case of some medical concepts, such as the general concept of DISEASE and the various individual disease concepts (such as PNEUMONIA, BREAST CANCER, SCHIZOPHRENIA, BORDERLINE PERSONALITY DISORDER, and so on) show the same "problems" presented by most common-sense concepts, as they can hardly be represented in terms of individually necessary and jointly sufficient conditions. Faced with the issues raised by the many attempts to find a traditional definition for the general concept of DISEASE (Amoretti 2015), some philosophers of medicine have thus proposed to regard the concept of DISEASE and those of individual diseases as non-classical ones. In this vein, on the grounds of the great variability among individual diseases, new theories based on family resemblances, prototypes or exemplars have been proposed (see e.g. Sadegh-Zadeh 2011, 2000, 2008, Lilienfeld and Marino 1995, 1999, Pickering 2013, 2016, McNally 2011).

In the case of family resemblances, there is no common feature that all individual diseases must have, but any two of them should share at least one feature. In the case of prototypes, there is a set of properties that represents the best instance of the disease category, that is an ideal and abstract construction of the general concept of disease, the prototype, to which any individual disease must approximate to some degree, sharing with it a goodly number of properties. In the case of exemplars, some individual diseases are regarded as particularly relevant, as the exemplars of the disease category, and thus all other diseases must exhibit a goodly number of their specific features.

These views are obviously different: embracing the family resemblances theory implies that there is no specific set of properties, determined by the prototype or the exemplars, that individual diseases must meet to some degree; the prototype is an abstract construction that doesn't need to correspond to any individual disease, while the 
exemplars are concrete members of the category. Nevertheless they are often conflated or muddled in the relevant literature.

For example, McNally (2011, p. 212) refers to Wittgenstein saying that "Examples of most useful concepts bear only a family resemblance to one another. Most have some overlapping attributes without sharing an essence present in every case"; but clearly he has in mind the prototype view, as he continues specifying that "The more attributes a given case has, the better an example it is of the concept". A similar confusion is made by Cooper (2007, p. 41), who mentions family resemblances saying that "While there need not be any one feature that all family members possess, any two members will be similar in a variety of ways"; however, she unpacks this idea through the exemplar view: "whether a condition counts as a mental disorder depends on its degree of resemblance to prototypical cases, such as schizophrenia and psychotic depression. Conditions that are sufficiently like these central cases get counted as disorders". Again, Sadegh-Zadeh (2008, p. 119) seems to conflates prototypes and exemplars claiming that "A concept determines a category $[\ldots]$ by exhibiting the relational structure of the category that is characterized by best examples, called prototypes, such that other category resemble them to different extents".

The above confusions can be partially explained by the fact that all three views offer a plausible way to deal with conceptual "typicality", that is the evidence that some instances of the general category of disease, namely some individual diseases, are regarded as more representative than others. Moreover, all three views agree that there is no set of properties shared by all and only individual diseases: no specific property is individually necessary and no fixed number of them is sufficient to characterize the general concept of DISEASE. On the contrary, overall similarities among different set of properties should encompass the absence of any particular shared property - such as, as it is often claimed, dysfunction (Boorse 1976, Wakefield 1992, 1999).

Many scholars adopting one of the above strategies do not attempt to better explicate the similarities relationship among individual diseases (Lilienfeld and Marino 1995, 1999); others think that fuzzy 
logic is the best, and possibly the only, way do the job (Seising and Tabacchi 2013, Sadegh-Zadeh 2000, 2008, 2011) - but, of course, some important alternatives to represent non-classical concepts have been proposed in the general literature, especially because fuzzylogic faces some unavoidable difficulties in handling typicality (on this aspect see Frixione and Lieto 2014a).

As sketched above, approaches based on family resemblances, prototypes, and exemplars have been used to characterize the general concept of DISEASE, but they seem particularly suited to handle the general concept of MENTAL DISORDER (Lilienfeld and Marino $1995,1999)$ as well as the various concepts of individual mental disorders. This more restricted class of medical concepts will be the focus of our present work.

The DSM (the Diagnostic and Statistic Manual of Mental Disorders), which is published by the American Psychiatric Association and represents a sort of "bible" for psychiatrists and scholars within the field of mental pathology, has in fact a merely descriptive approach: it rarely incorporates theoretical information regarding the causes of individual mental disorders, and classifies them using a list of operational diagnostic criteria. As a consequence, and somehow differently to what usually happens with individual somatic diseases included in ICD (the International Classification of Disease), individual mental disorders are typically identified not by their etiology or underlying pathological cause (a few exceptions being, for example, the different types of neurocognitive disorders), but through their syndromes, that is through a catalogue of their characterizing symptoms and signs; in most cases none of them is individually necessary and no fixed number of them is sufficient to determine membership to a certain individual disorder category. Moreover, in most cases these syndromes are not supposed to be reified, as to correspond to some kind of entity or mechanism (such as an underlying dysfunction).

Let's see, for instance, an oversimplified version of the diagnostic criteria for schizophrenia and borderline personality disorder given by the DSM-5. Criterion A for schizophrenia states:

A. Two (or more) of the following, each present for a significant portion of time during a 1 -month period (or less if successfully treated). At least one of these must be (1), (2), or (3): 
1. Delusions.

2. Hallucinations.

3. Disorganized speech (e.g., frequent derailment or incoherence).

4. Grossly disorganized or catatonic behavior.

5. Negative symptoms (i.e., diminished emotional expression or avolition) (American Psychiatric Association 2013, p. 99, our italics).

Similarly, but even more explicatory, borderline personality disorder is characterized as follows:

A pervasive pattern of instability of interpersonal relationships, self-image, and affects, and marked impulsivity, beginning by early adulthood and present in a variety of contexts, as indicated by five (or more) of the following:

1. Frantic efforts to avoid real or imagined abandonment. [...]

2. A pattern of unstable and intense interpersonal relationships characterized by alternating between extremes of idealization and devaluation.

3. Identity disturbance: markedly and persistently unstable self-image or sense of self.

4. Impulsivity in at least two areas that are potentially self-damaging (e.g., spending, sex, substance abuse, reckless driving, binge eating). [...]

5. Recurrent suicidal behavior, gestures, or threats, or self-mutilating behavior.

6. Affective instability due to a marked reactivity of mood (e.g., intense episodic dysphoria, irritability, or anxiety usually lasting a few hours and only rarely more than a few days).

7. Chronic feelings of emptiness.

8. Inappropriate, intense anger or difficulty controlling anger (e.g., frequent displays of temper, constant anger, recurrent physical fights).

9. Transient, stress-related paranoid ideation or severe dissociative symptoms (American Psychiatric Association 2013, p. 663, our italics).

It is easy to see that there are many different ways to meet the requirements of schizophrenia or bipolar personality disorders stated above, and it is of course possible that members of these categories have no characteristics in common. For example, Galatzer-Levy and Bryant (2013) recently calculated that there are 636,120 ways to meet the requirements of the concept of post-traumatic stress disorder.

The operational criteria, introduced in DSM-III (1982), were meant to replace what psychiatrists dub as "prototypes", that is short descriptions of paradigmatic cases that would serve as standards of comparison to evaluate and diagnose any single patient. Here, as an 
example, the category of schizophrenic reactions according to DSMI (1952):

It represents a group of psychotic reactions characterized by fundamental disturbances in reality relationships and concept formations, with affective, behavioral, and intellectual disturbances in varying degrees and mixtures. The disorders are marked by strong tendency to retreat from reality, by emotional disharmony, unpredictable disturbances in stream of thought, regressive behavior, and in some, by a tendency to deterioration, (American Psychiatric Association 1952, p. 26).

Even if the operational structure of DSM-5 coincides neither with the prototype nor the exemplar views as they are developed by cognitive psychologists, it may still suggest to incorporate some features of these approaches in the representations of the various concepts of individual mental disorders (such as, SCHIZOPHRENIA, BORDERLINE PERSONALITY DISORDER, MAJOR DEPRESSION, etc.) as well as the general concept of MENTAL DISORDER, as like non-classical concepts they cannot be possibly defined through necessary and sufficient conditions, and clearly exhibit prototypical effects.

In order to address this problem from a computational perspective, we have analyzed the field of logic-oriented knowledge representation systems and, in particular, the class of formalisms known as formal ontologies. We provide below a brief overview of this class of systems by showing that, also in this artificial context, we face the problem of representing typical or "non-classical" information of medical concepts.

\section{Formal Ontologies and Common-Sense Representations}

In the last decades the problem of concept representation received a great deal of attention within the field of artificial intelligence (AI), and in particular in knowledge representation, due to its relevance for semantic technologies and for the development of formal ontologies.

In the AI tradition, an ontology is "an engineering artifact, constituted by a specific vocabulary used to describe a certain reality, plus a set of explicit (axiomatic) assumptions regarding the intended meaning of the vocabulary words" (Guarino 1998). The representation 
languages adopted for the development of formal ontologies stemmed from the tradition of so-called structured inheritance semantic networks - the first system in this line of research was KLONE (Brachman and Schmolze 1985). These formalisms are known today as description logics (DLs), and the main formal ontological languages such as OWL and OWL 2 belong to this class. The main constructs of such languages are concepts (or classes), roles (or properties), and individuals.

DLs are logical systems (usually, they are subsets of first order predicate calculus). They can perform a series of important automatic inferences, such as categorization (the process of attributing a specific individual as a member to a class), classification (the process through which new class-subclass relations are inferred) and consistency checking (the process of testing the logical coherence of a given ontology).

As logical systems, DLs have a model theoretic, Tarskian style semantics associated to them (Horrocks et al. 2003). This fact is a symptom of a problem: Tarskian semantics is fully compositional, and typicality effects are hard to accommodate with compositionality (Fodor 1981). Indeed, DLs do not allow the representation of concepts in prototypical terms (on this aspect see Frixione and Lieto, 2012). DLs allow the representation of concepts exclusively in terms of sets of necessary and/or sufficient conditions. This is a severe drawback from the standpoint of the representation of many classes of concepts.

In particular, this strong bias towards the representation of concepts in terms of necessary and/or sufficient conditions alone is also a problem in the field of medical ontologies. Most of them, indeed, (including SNOMED, the largest biomedical ontology currently available: http://www.ihtsdo.org/snomed-ct) are conditioned by the adoption of formalisms that do not allow to represent concepts in typical terms.

This possibility should be of crucial importance for representing both such general concepts as DISEASE or MENTAL DISORDER, and the concepts of individual diseases and mental disorders. Consider for example the concepts of individual mental disorders. In DSM-5 they are characterized in terms of syndromes and operational criteria. However, at the level of specific mental disorders, it 
is often impossible to individuate sets of symptoms and criteria that are individually necessary and/or jointly sufficient to determine membership.

\section{Some Preliminaries of a Case Study: The Schizophrenia Spectrum}

As a preliminary step to prove our hypothesis that the general concept of MENTAL DISORDER and (most of) the various concepts of individual mental disorders - as they are currently described and categorized by DSM-5 - should be treated as non-classical ones is preliminary tested by developing an ontology based on the OWL-DL (Ontology Web Language Description Logic) dialect. Some important remarks must be done here.

Despite the fact that there already are formal ontologies dealing with mental disorders (SNOMED is such an example), we decided to develop a new one trying to overcome some of their potential limitations. In particular, with respect to the existing taxonomies of mental disorders, that are typically part of larger representation systems and must thus meet their criteria and general principles, we are currently building a representation that aims to be independent to previous ones and closer to the DSM-5 nosology and rationales (having also well clear in mind what are the main limits and problems of the Statistical Manual). We believe it is a necessary stage in order to verify and evaluate the exact limits of a classical approach to the formal representation of the concepts of individual mental disorders, as we suspect that some problems encountered by the already available formal ontologies might be due to an oversimplification of the structure and rationales of DSM-5 descriptive nosology, which is syndrome based and criterial.

On this respect, we take seriously the DSM-5 definition of the concept of MENTAL DISORDER, according to which a mental disorder is primarily a syndrome, that is a set of symptoms and signs. This means, for example, that the classes of Mental_Disorder and Symptom must be linked through an appropriate property (it must be remembered that, in OWL terminology, properties correspond to roles, or two place relations). Making the relationships between 
mental disorders and pattern of symptoms explicit might also help to clear out some classification disputes about where to place some controversial mental disorders among DSM-5 chapters.

Moreover, even if the DSM-5 definition of the concept of MENTAL DISORDER requires a dysfunction being in place, there is also the widespread conviction that syndromes should not be reified. The possibility to discover that basic dimensions of functioning, and thus dysfunctioning, cut across traditional syndrome-based diagnostic categories is actually envisaged - as the NIMH Research Domain Criteria (RDoC) project seems to corroborate. This means, for example, that the class of Mental_Disorder must be conceived in non realist terms and the concept of MENTAL DISORDER clearly distinguished from the concept of DISEASE.

Broadly speaking, the rationale we have followed to build our DSM5 compliant ontology can be summarized in 4 steps, as shown in Figure 1 below:

1. Identification of main concepts;

2. Formalization of classes and properties;

3. Implementation;

4. Comparison between symptoms and evaluation (i.e. modeling decision about the taxonomical position and the related axioms that need to be added).

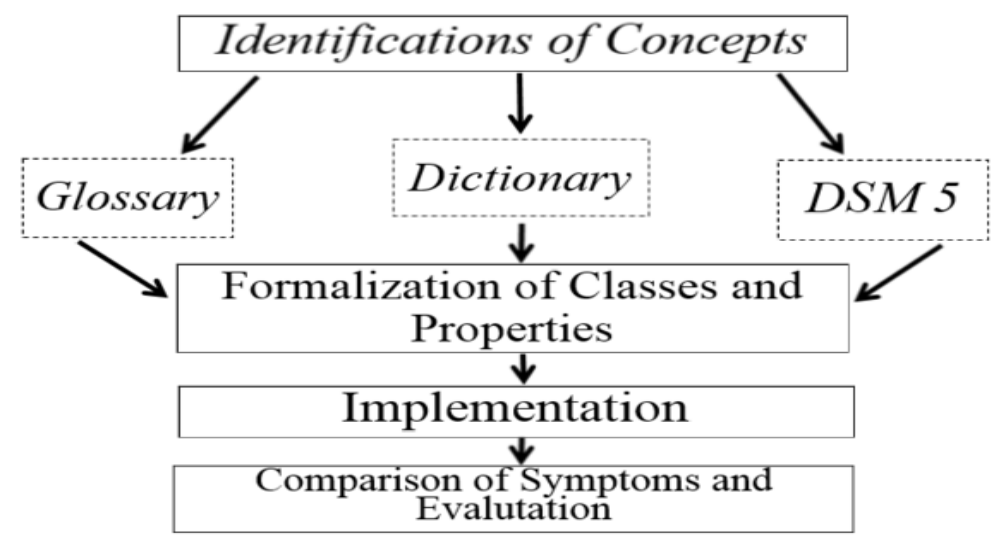

Fig. 1. The process of building a DSM-5 compliant ontology. 
The goal of the first phase was identifying, organizing and structuring all the main concepts of the domain by using an abstract model, e.g. graphs or schemes. Initially, we focused on the chapter of Schizophrenia Spectrum only, and defined relevant concepts and properties through a glossary or dictionary written in natural language. Afterwards, with the second phase we used description logics to formalize all the concepts and properties previously identified and thus to obtain the adequate terminological domain knowledge. The third phase aimed at encoding and implementing a formal ontology using Protégé, a widespread ontology editor developed at the Stanford University (http://protege.stanford.edu/). In the fourth and last phase we compared various symptoms among ontologies and different disorders. Moreover, the process of evaluation can be also driven in parallel with the previous three steps. 

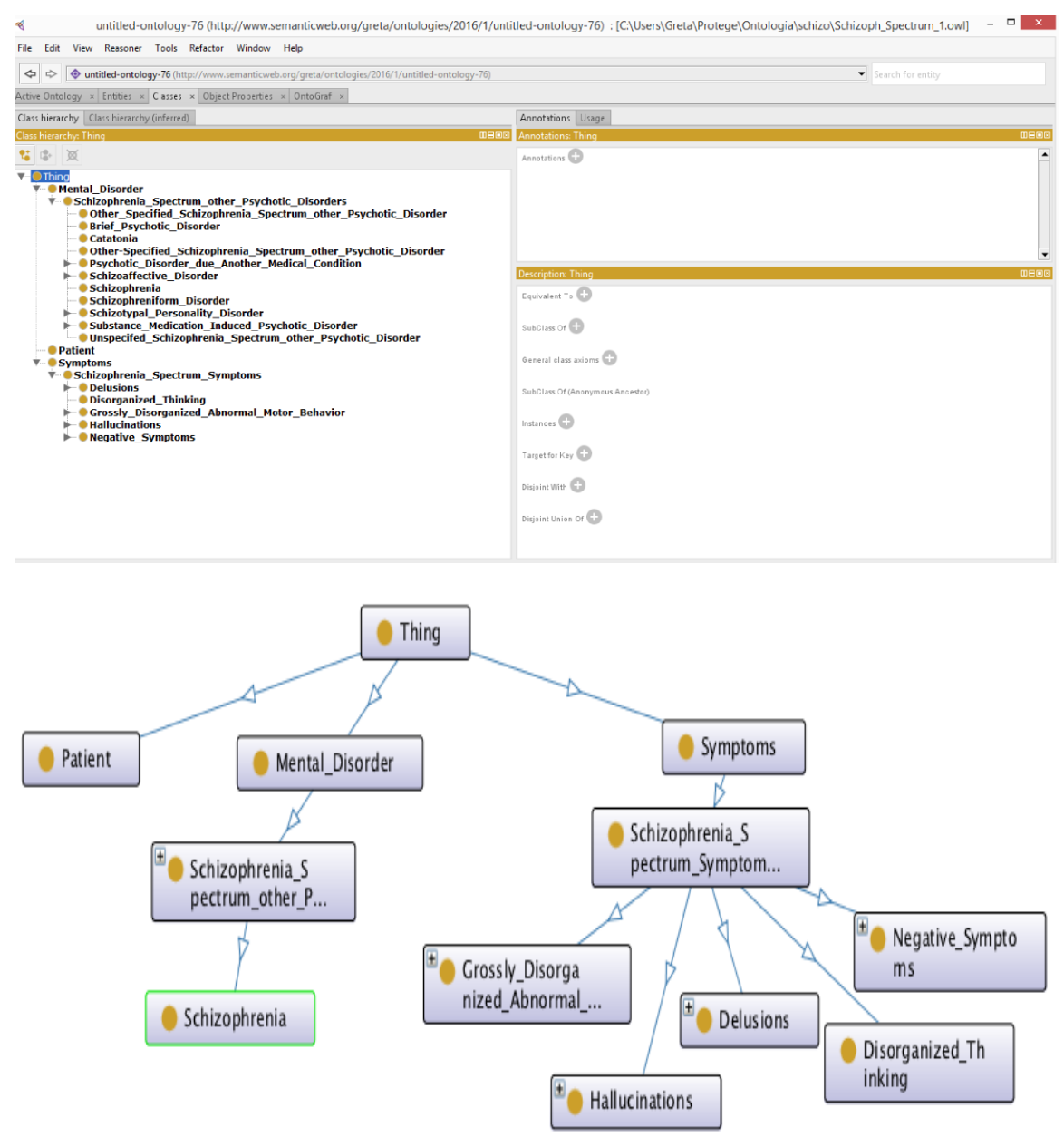

Fig. 2. Top Level of the Schizophrenia Spectrum Ontology.

The Schizophrenia_Spectrum ontology that we have developed is currently composed by 58 classes, 5 properties and 191 axioms. As already mentioned, the ontology has been developed by adopting the OWL-DL (Ontology Web Language-Description Logic) dialect.

The three main classes are Mental_Disorder, Patient and Symptom. The top level of the ontology, which focuses on the various classes of the Schizophrenia Spectrum category and the associated symptoms, is shown in Figure 2 above.

The top-level classes chosen by the adopted modeling not only allow that each mental disorder might be identified through quite different 
set of symptoms (as it is clearly demanded by the DSM-5 diagnostic criteria), but also address comorbidity (a phenomenon which is still common in DSM-5 meaning that each patient showing a certain set of symptoms might be diagnosed with more than one mental disorder).

The class of Patient allows to model many different patient instances, which is useful in order to include personal information regarding individuals (such as age, sex, gender, ethnicity, etcetera).

Finally, the class Symptom currently contains the following main subclasses, which have been built in accordance with the DSM-5 criteria: Delusions, Disorganized Thinking, Grossly Disorganized Abnormal Motor Behavior, Hallucinations, Negative Symptoms.

The class of Symptom and its subclasses are disjointed from the Schizophrenia Spectrum other Psychotic Disorder and its subclasses as this guarantees the separation from symptoms that involves other mental disease.

The current version of the ontology (which is still subject to revisions and extensions) is available in a navigable format at: http:// www.di.unito.it/ lieto/Schizophrenia Spectrum.html. Even if we developed a formal ontology which is more DSM-5 compliant than others (for instance, Ceusters and Smith 2010), as we predicted it is still unable to handle the representation and reasoning of commonsense cues.

\section{A proposal: A Hybrid Architecture}

In this perspective - given the fact that the concepts of MENTAL DISORDER and individual mental disorders clearly exhibit typicality effects that cannot be handled with traditional, purely compositional, representational systems - we propose to integrate typicality effects in computational representations of concepts. More precisely, we focus on prototypical and exemplar based approaches, and propose to combine them in a hybrid model. (For the time being, we do not take into consideration here theory-theory approaches, since them are in some sense more vaguely defined if compared to the other two positions.) 
Following the approach proposed in Frixione and Lieto (2013, 2014b) and preliminary tested in Lieto et al. (2017), we propose a hybrid architecture (see Figure 3 below) combining a "classical" component (in which concepts are represented, as far as it is possible, in terms of necessary and/or sufficient conditions) with a "typicality-oriented" component, allowing both prototype and exemplarbased representations.

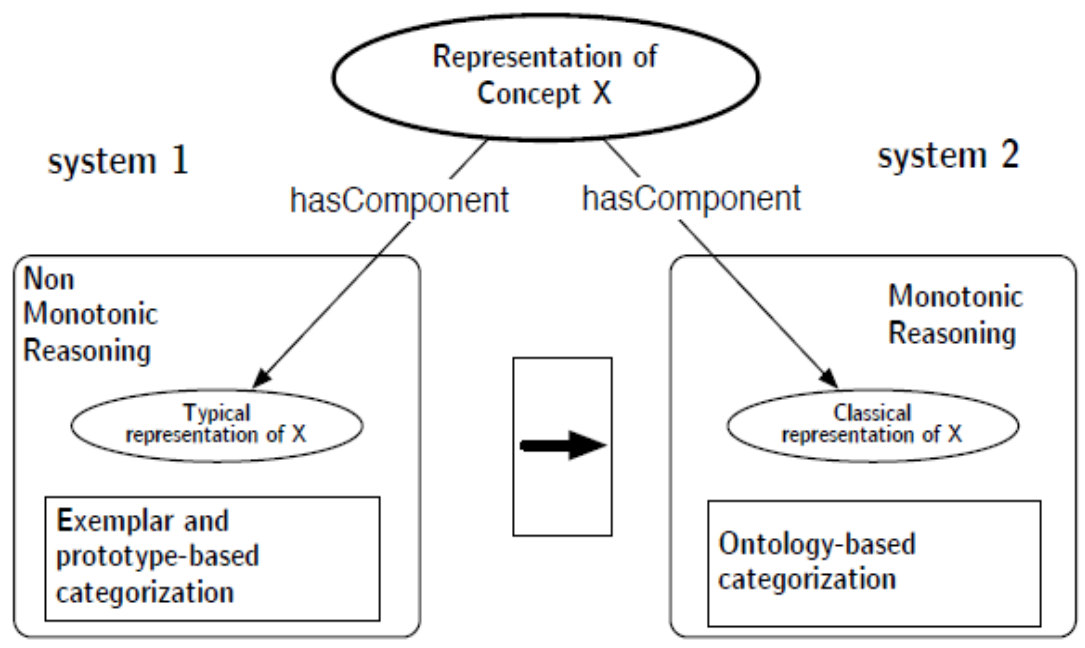

Fig. 3. The conceptual architecture we propose.

The "classical" component is demanded to some standard ontological formalism, such as DLs; the "typicality-oriented" component to a conceptual space, where conceptual spaces are a geometric framework for knowledge representation proposed by Peter Gärdenfors (2014).

In a conceptual space concepts are described in terms of a number of quality dimensions. In some cases, such dimensions are directly related to perception; examples could be temperature, weight, brightness, pitch. In other cases, dimensions can be more abstract in nature. To each quality dimension is associated a geometrical (topological or metrical) structure.

The central idea behind this approach is that the representation of knowledge can take advantage from the geometrical structure of the 
spaces. Instances (or exemplars) are represented as points in a space, and their degree of similarity can be calculated in a natural way according to some suitable distance measure. Concepts correspond to regions, and regions with different geometrical properties correspond to different kinds of concepts. Prototypes and typicality effects have a natural geometrical interpretation: a prototype corresponds to the geometrical centre of the region representing a concept (provided that the concept corresponds to a convex region). Thus, given a concept, a degree of centrality can be associated to each point that falls within the corresponding region. This degree of centrality can be interpreted as a measure of its typicality. Conversely, given a set of $n$ prototypes represented as points in a conceptual space, a tessellation of the space in $n$ convex regions can be determined in the terms of the so-called Voronoi diagrams. An example is shown in Figure 4, where the center of each region corresponds to the prototype of a given concept, and where different exemplars can be represented as points in a conceptual region. The similarity between exemplars, or between prototypes and exemplars is obtained by calculating the metric distances in the underlying space.

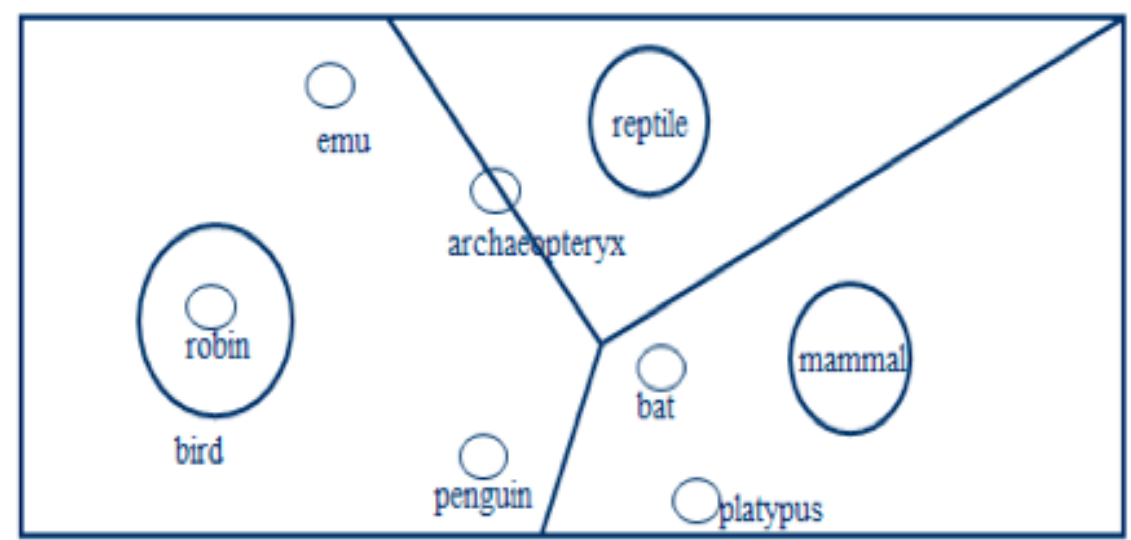

Fig. 4. An example of the Voronoi tessellation of a conceptual spaces (from Gardenfors and Williams 2001).

In sum, the appeal of conceptual spaces consists in the fact that they provides a natural way of representing typicality effects, and that their geometrical structure provides a natural way of calculating the semantic relations between concepts, prototypes and exemplars in 
terms of metrical distance. In general, conceptual spaces seem to provide a better framework for modeling typicality effects in artificial system if compared to both standard symbolic systems and connectionist architectures - on this aspect see Lieto, Chella and Frixione (2017).

Considering the concepts of MENTAL DISORDER and individual mental disorders an hybrid architecture as the one described above would result particularly useful. On the one hand, the "classical" component - demanded to the ontology we developed and described in the above section - would allow us to make important inferences and comparisons between individual mental disorders. Moreover, it would be needed to clearly represent some individual mental disorders that seem to be characterized by necessary (and sufficient) conditions (their medical or substance aetiology or some specific symptoms). For example, Bulimia nervosa seems to be characterized by necessary and sufficient conditions, as shown by its diagnostic criteria below:

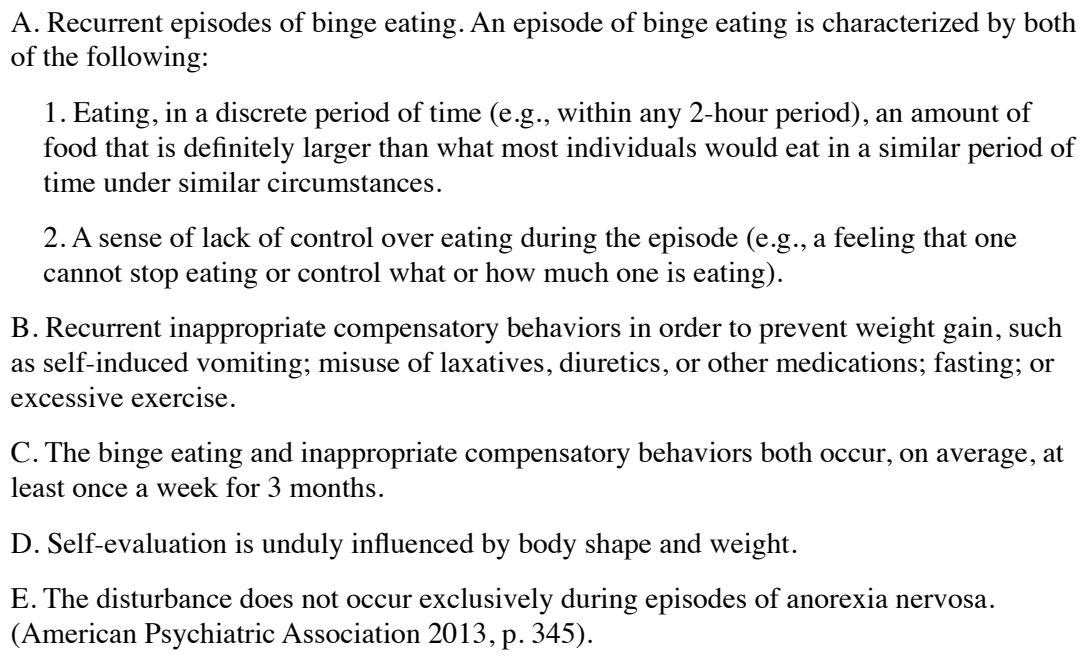

B. Recurrent inappropriate compensatory behaviors in order to prevent weight gain, such as self-induced vomiting; misuse of laxatives, diuretics, or other medications; fasting; or excessive exercise.

$\mathrm{C}$. The binge eating and inappropriate compensatory behaviors both occur, on average, at least once a week for 3 months.

D. Self-evaluation is unduly influenced by body shape and weight.

E. The disturbance does not occur exclusively during episodes of anorexia nervosa.

(American Psychiatric Association 2013, p. 345).

Alternatively, Major neurocognitive disorders are characterized by similar syndromes and differentiated by their underline pathological cause (e.g., Alzheimer's disease, Frontotemporal lobar degeneration, Lewy body disease, Vascular disease, Traumatic brain injury, HIV infection, Prion disease, Parkinson's disease, Huntington's disease), which is thus a necessary condition for their diagnosis. 
Also the general concept of MENTAL DISORDER, at least its theoretical and "conceptually clean" version - as the one stated in the Introduction of DSM-5 - may allow for necessary (and sufficient) criteria, such as the dysfunction requirement:

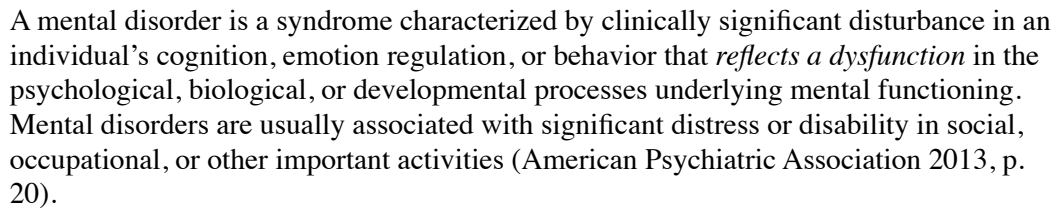

On the other hand, the "typicality-oriented" component would be necessary to deal with typicality effects and handle all those individual mental disorders, such as those listed in the chapter "Schizophrenia Spectrum and Other Psychotic Disorders", that are not characterized by necessary and sufficient conditions. Moreover, as far as the general concept of MENTAL DISORDER is concerned, the "typically-oriented" component would be useful to represent its common sense or practical version, which is much needed to guide us in distinguishing between health and pathological conditions in most of ordinary situations (Amoretti, Frixione, Lieto 2017).

On this respect, we shall try to develop a conceptual space with a number of quality dimensions able to identify the prototype of mental disorder as well as the relevant exemplars. Some candidates for the qualitative dimensions might be related to the duration of symptoms, their clinical significance, their functional dimensions, and so on. Such as geometric framework would constitute the "non-classical" component of our architecture. Then, we shall also try to represent the various concepts of individual mental disorders within such as conceptual space and evaluate their positions, as well as their degree of typicality.

\section{Concluding remarks}

To sum up, we exposed the problems raised by conceptual "typicality" to the classical theory of concepts, focusing on the general concept of MENTAL DISORDER and the various concepts of individ- 
ual mental disorders as described by DSM-5. Then, we summarized one important issue faced by description logics in representing medical knowledge: as they are associated to a model theoretic, Tarskian style semantics, they prove to be unable to represent concepts in prototypical terms. To reinforce this conclusion, we build an ontology specifically suited to represent the general concept of MENTAL DISORDER and (most of) the various concepts of individual mental disorders. Despite being more DSM-5 compliant than other ontologies, our formalism was still unable to handle typicality effects. We thus proposes a hybrid approach combining a "classical" component (in which concepts are represented in terms of necessary and/or sufficient conditions) with a "typicality-oriented" component, allowing both prototype and exemplar-based representations.

In order to develop such a hybrid architecture, the next step would be defining a suitable number of quality dimensions able to identify the prototype of the general concept of MENTAL DISORDER and its relevant exemplars (such as SCHIZOPHRENIA, BORDERLINE PERSONALITY DISORDER, or MAJOR DEPRESSION). One possible application of this integration would be the realization of an artificial system that, given a set of typical traits characterizing the different symptoms, would be able to provide the identification of the corresponding mental disorder.

\section{References}

American Psychiatric Association, 1952. Diagnostic and Statistical Manual. Mental Disorders, Washington, DC: American Psychiatric Association.

American Psychiatric Association, 2013. Diagnostic and Statistical Manual of Mental Disorders, Fifth Edition: DSM-5. Washington, DC: American Psychiatric Publishing.

Amoretti, M.C., 2015. Filosofia e medicina. Pensare la salute e la malattia. Roma: Carocci.

Amoretti, M.C., M. Frixione, A. Lieto, 2017. "The benefits of prototypes: The case of medical concepts". Reti, Saperi e Linguaggi. Italian Journal of Cognitive Sciences, 4(1): 97-114.

Boorse, C., 1976. "What a theory of mental health should be", Journal for the Theory of Social Behaviour, 6: 61-84.

Brachman, R, J.G. Schmolze, 1985. "An overview of the KL-ONE knowledge representation system”, Cognitive Science, 9: 171-216.

Ceusters, W, B. Smith, 2010. "Foundations for a realist ontology of mental disease", Journal of Biomedical Semantics, 1(1): 10.

Cooper, R., 2007. Psychiatry and Philosophy of Science. Montreal: McGill-Queen's University Press. 
Fodor, J., 1981. "The present status of the innateness controversy". In: J. Fodor, Representations, Cambridge, MA: MIT Press.

Frixione, M., A. Lieto, 2012. "Representing Concepts in Formal Ontologies: Compositionality vs Typicality Effects", Logic and Logical Philosophy, 21: 391-414.

Frixione, M., A. Lieto, 2013. "Dealing with Concepts: From Cognitive Psychology to Knowledge Representation", Frontiers in Psychological and Behavioural Science, 2(3): 96-106.

Frixione, M., A. Lieto, 2014a. "Concepts, Perception and the Dual Process Theories of Mind", Baltic International Yearbook of Cognition, Logic and Communication, 9: 1-20.

Frixione, M., A. Lieto, 2014b. "Towards an Extended Model of Conceptual Representations in Formal Ontologies: A Typicality-Based Proposal", Journal of Universal Computer Science, 20(3): 257-276.

Galatzer-Levy, I.R., R.A. Bryant, 2013. "636,120 Ways to Have Posttraumatic Stress Disorder", Perspectives on Psychological Science, 8(6): 651-662.

Gärdenfors, P., 2014. The Geometry of Meaning. Semantics Based on Conceptual Spaces. Boston: MIT Press.

Gärdenfors, P, M.-A. Williams, 2001. "Reasoning about Categories in Conceptual Spaces", Proc. IJCAI 2001, 385-392.

Guarino, N., 1998. "Formal ontology in information systems", Proc. First Int. Conf. on Formal Ontologies in Information Systems (FOIS'98), June 6-8, Trento, Italy, 46. Amsterdam: IOS press.

Horrocks, I., P.F. Patel-Schneider, F. Van Harmelen, 2003. "From shiq and rdf to owl: The making of a web ontology language", Web Semantics: Science, Services and Agents on the World Wide Web, 1(1): 7- 26 .

Lilienfeld, S.O., L. Marino, 1995. "Mental disorder as a Roschian concept: A critique of Wakefield's 'harmful dysfunction' analysis", Journal of Abnormal Psychology, 104 (3): 411-420.

Lilienfeld, S.O., L. Marino, 1999. "Essentialism revisited: Evolutionary theory and the concept of mental disorder", Journal of Abnormal Psychology, 108 (3): 400-411.

Lieto, A., Radicioni, D. P., \& Rho, V. (2017). "Dual PECCS: a cognitive system for conceptual representation and categorization". Journal of Experimental \& Theoretical Artificial Intelligence, 29(2), 433-452.

Lieto A., Chella, A., Frixione M., (2017). "Conceptual Spaces for Cognitive Architectures: A Lingua Franca for Different Levels of Representation”, Biologically Inspired Cognitive Architecture, 19, 1-9.

Machery, E., 2010. Doing without Concepts. Oxford: Oxford University Press.

McNally, R., 2011. What is Mental Illness? Cambridge, MA: Belknap Press of Harvard University Press.

Murphy, G., 2002. The Big Book of Concepts. Cambridge, MA: MIT Press.

Pickering, N., 2013. "Extending disorder: essentialism, family resemblance and secondary sense", Medicine, Health Care and Philosophy, 16(2): 185-195.

Pickering, N., 2016. "Disease, Variety, Disagreement, and Typicality. Advantage Roschian Concepts?" Philosophy, Psychiatry and Psychology, 23(1):17-31.

Rosch, E., 1975. "Cognitive representation of semantic categories", Journal of Experimental Psychology, 104: 573-605.

Rosch, E., C.B. Mervis, 1975. "Family resemblances: Studies in the internal structure of categories", Cognitive psychology, 7(4): 573-605.

Sadegh-Zadeh, K., 2000. "Fuzzy Health, Illness, and Disease", Journal of Medicine and Philosophy, 25(5): 605-638.

Sadegh-Zadeh, K., 2008. "The Prototype Resemblance Theory of Disease", Journal of Medicine and Philosophy, 33(2): 106-139.

Sadegh-Zadeh, K., 2011. Handbook of Analytic Philosophy of Medicine. New York: Springer.

Seising, R., M. Tabacchi, 2013. Fuzziness and Medicine. Dordrecht: Springer. 
Wakefield, J.C., 1992. "The concept of mental disorder. On the boundary between biological facts and social values", American Psychologist, 47: 373-388.

Wakefield, J.C., 1999. "Evolutionary versus prototype analyses of the concept of disorder", Journal of Abnormal Psychology, 108(3): 374-399.

Wittgenstein, L, 1953. Philosophische Untersuchungen/Philosophical Investigations. Oxford: Blackwell. 